\title{
La política de innovación en Colombia ${ }^{1}$
}

Iván Montenegro

COLCIENCIAS

imontenegrotrujillo@gmail.com

El propósito de este artículo es sustentar la centralidad de una efectiva política pública de innovación, ciencia y tecnología con miras a contribuir al desarrollo productivo y la inclusión social. Para ello se adopta un marco conceptual robusto que, partiendo de la triple falla (mercado, sistema y Estado), sustenta componentes clave de la política como son la gobernanza y la focalización en ciencia, innovación y desarrollo productivo. Hay conciencia de que en el mundo existe un conjunto de métodos, modelos y técnicas que facilitan la consecución de objetivos aparentemente contradictorios. Entre los retos planteados, cabe mencionar, en los niveles nacional y territorial, el apoyo de la ciencia, la innovación y el desarrollo productivo al posconflicto colombiano y del sector informal de la economía.

Palabras clave: innovación, política pública, desarrollo productivo, gobernanza, posconflicto

The purpose of the article is to support the main role of an effective public policy of innovation, science and technology, oriented to contribute to productive development and social inclusion. To this objective, a strong conceptual framework is adopted that emerges from the threefold failure: market, system and State. It supports policy's key components such as: governance and focus on science, innovation, and productive development. There is awareness that in the world there exists a set of methods, models and techniques that facilitate the achievement of seemingly contradictory objectives. Among the challenges presented, note is made of the support of science, innovation, and productive development to post-conflict Colombian nation and territory and to the economy's informal sector.

Keywords: innovation, public policy, productive development, governance, post-conflict

1. Este artículo está basado en la ponencia presentada como parte de la mesa magistral «Políticas públicas de promoción de la innovación en América Latina», durante el I Congreso Internacional de Ciencias de la Gestión: Gestión de la Innovación e Innovación en la Gestión, desarrollado del 29 de setiembre al 2 de octubre de 2015 en la Pontificia Universidad Católica del Perú por el Departamento Académico de Ciencias de la Gestión y la Facultad de Gestión y Alta Dirección. 


\section{Introducción}

El abordaje inicial del tema es identificar el problema central de la innovación en Colombia, definido como las débiles condiciones y vínculos entre los subsistemas y entre los actores del sistema nacional de ciencia, tecnología e innovación para facilitar la innovación. Lo anterior tiene en cuenta las limitaciones y efectos negativos del uso, sin suficiente reflexión contextual, del enfoque conceptual del sistema nacional de innovación, que es acompañado de una tradicional visión de empuje del conocimiento que prepondera hasta el presente (Velasco, 2015).

Entre las principales causas del estancamiento de la innovación y de la investigación es preciso mencionar el profundo legado de la constitucionalidad confesional del siglo XIX en el plano de la cultura y, como un caso particular del peculiar sincretismo colombiano de lo moderno y premoderno, con relación al contexto latinoamericano, se debe tener en cuenta la asimilación superficial de la racionalidad de la ciencia moderna a la manera de una «revelación» de índole religiosa que dificulta que las ciencias contribuyeran a la modernidad en Colombia (Villaveces Cardozo, 1991). Otra causa es el acceso incipiente a la temática científica para amplios sectores de la población en el pasado, si bien el acceso ha mejorado en tiempos recientes. Una causa más cercana del precario avance de la innovación es el tipo de formación de la tecnocracia pública y privada con su énfasis en una radical visión neoclásica de preponderancia del mercado.

Uno de los efectos del problema central es el bajo nivel de productividad del sector productivo colombiano, el cual, en términos de productividad laboral y con referencia a Estados Unidos en 2013, muestra una productividad laboral nacional promedio del $22.69 \%$, variando desde el $55 \%$ para minas y canteras, y $18 \%$ en la industria manufacturera, hasta el $9 \%$ de agricultura, ganadería, silvicultura, caza y pesca (Consejo Privado de Competitividad, 2015).

Se justifica mencionar la necesidad de explorar la relación entre el escaso avance de la innovación y el muy elevado nivel de inequidad en el ingreso y la riqueza en Colombia, por las particulares características de rentismo del decil más rico de la población, que configuran a Colombia, con la aplicación de la metodología de Piketty, como uno de los países más inequitativos de la región y del mundo (Londoño, 2013).

La inversión público-privada en innovación se justifica en su impacto positivo para el aumento de la productividad y el empleo (Crespi, 2010; Arbeláez \& Parra, 2011). Aquella podría, bajo una serie de supuestos, contribuir al crecimiento de la equidad en la riqueza, el ingreso y el empleo, y generar un primer círculo virtuoso entre la inversión en investigación y desarrollo (I\&D), el incremento de la productividad y la elevación del nivel de competitividad, lo cual posibilita a su vez una mayor inversión en I\&D.

Pero, en la coyuntura crítica que enfrenta Colombia, en el proceso de construcción de la paz, luego de un conflicto de cerca de 60 años, la innovación y la ciencia pueden contribuir, en sincronía con otras actuaciones público-privadas y reformas institucionales en lo político y económico, a una prosperidad inclusiva (Acemoglu \& Robinson, 2012), con la posibilidad de alimentar un circulo virtuoso de mayor escala en el que las instituciones políticas inclusivas fortalecen instituciones económicas inclusivas y viceversa. Este planteamiento se apoya en diversos estudios acerca del positivo impacto de la construcción de paz en Colombia (DNP, 2016). 


\section{Marco conceptual}

\subsection{Fallas de mercado}

El punto de partida es el reconocimiento, con una perspectiva amplia, de que el mercado falla en tanto no induce el aprovechamiento máximo de las oportunidades actuales y que falla también en su ampliación (Crespi, Fernández-Arias \& Stein, 2014). Con relación al primer tipo de fallas, están aquellas que se expresan en la escasez de insumos colectivos -infraestructura, formación de personal, inversión en I\&D- que justifican la actuación del Estado para proveerlos y/o coordinar a los agentes. Otra falla se refiere a la incapacidad para la coordinación de los actores con el fin de realizar inversiones cuya escala requiere de ella; y también falla el mercado cuando no induce un aprendizaje de la experiencia, debido a que este no puede, en principio, ser aprovechado por las empresas debido a que, por ejemplo, el personal capacitado puede movilizarse hacia los competidores (externalidades dinámicas).

En cuanto a las fallas de ampliación de oportunidades (Chang, 2011), se generan debido a que el mercado induce a la actuación en el corto y mediano plazo, y no al aprovechamiento de ellas en el largo plazo a través de la identificación de sectores o actividades prioritarias que, además de ser beneficiosas en el presente, se constituyen hacia el futuro sobre la base de capacidades para una ulterior diversificación hacia productos muy atractivos (complejos).

En cuanto a investigación y desarrollo, una de las principales pruebas de que el mercado falla en inducir a una inversión suficiente en I\&D es que, luego del último cuarto de siglo en Colombia — país que aplica el enfoque del Consenso de Washington-, dicha inversión, como proporción del PIB, equivale al 44\% de la inversión promedio en América Latina y a una décima parte de aquella de la OCDE.

Con relación a las fallas de mercado en $I \& D$, es preciso mencionar, en primer lugar, que una falla de mercado implica que el beneficio social neto de las actividades de ciencia, tecnología e innovación (ACTI) difiere del beneficio privado neto percibido por los agentes del mercado que controlan estas actividades; es decir, estas generan externalidades que pueden beneficiar o imponer costos a otros agentes que no han incidido en generarlas.

En segundo lugar, las fallas típicas son las de "apropiación del conocimiento» y las de "coordinación». Con relación a las primeras, hay que tener presente el caso de la investigación básica, en la que, además de la alta probabilidad de difusión pública de sus resultados y su menor probabilidad de uso en nuestros países debido al precario nivel de alfabetización científica de la mayor parte de la población, se agregan otros hechos como el explicable escaso interés del empresariado y su alto riesgo, todo lo cual justifica que sea el Estado el que asuma su realización. Cuando se trata de investigación más aplicada, se producen externalidades positivas que generan una inversión social no óptima en esta materia.

En el caso de la innovación, las fallas de apropiación se presentan cuando el escaso avance y/o la parálisis en el proceso innovador se debe también al temor de copia que tienen los pioneros cuando "descubren" los costos y los procesos de diversificación basados en innovación (Hausmann \& Rodrick, 2003), lo que justifica una actuación del Estado para lograr una inversión social óptima en innovación o apropiación tecnológica. 
Las "asimetrías de información» se constituyen en otro tipo de fallas de mercado cuando, debido a la escasa información de las agencias financieras tradicionales, no es posible para ellas distinguir en las empresas los problemas de liquidez de los de factibilidad — sobre todo en las PYMES y en los emprendimientos de base tecnológica-, lo que tiene como efecto que se mantengan en niveles muy bajos de productividad por el mínimo avance de sus capacidades tecnológicas. A lo anterior, se agrega el problema de la incertidumbre inherente a los procesos de innovación. Estos problemas justifican la implementación de la banca de desarrollo para apoyar la innovación y la actuación de intermediarios — brokers tecnológicos-, respectivamente.

Otro problema relacionado con la información es el hecho de que el mercado no induce el logro de conocimiento e información de mediano y largo plazo en cuanto a temas de investigación y de tendencias científico-tecnológicas, lo cual justifica la actuación estatal para proveer con bienes públicos estudios de prospectiva tecnológica y científica, y hojas de ruta tecnológicas.

\subsection{Fallas de coordinación}

Las fallas de coordinación se presentan cuando, a causa de ellas, las ACTI y las empresas innovadoras relacionadas entre sí no prosperan, ya que el mercado por sí solo no lo hace. Tal es el caso de Costa Rica y su sector de implementos médicos complejos, que requería de la prestación de servicios de esterilización para poder evolucionar hacia la producción de implementos médicos más sofisticados, lo cual requirió de una política estatal de atracción de inversión extranjera directa (IED) para la prestación de dichos servicios de esterilización, con lo cual la producción más compleja se logró al contar con esos servicios clave (Crespi, Fernández-Arias \& Stein, 2014).

Las fallas de coordinación entre empresas — generalmente PYMES - suceden cuando se requiere asumir altos costos fijos que generan muy bajos costos marginales en cuantiosas inversiones que son inalcanzables para las empresas individuales, pero que son factibles cuando se logra la coordinación para la participación conjunta de aquellas mediante el apoyo del Estado (ver tabla 1).

\section{Tabla 1: Fallas de mercado en ciencia, tecnología e innovación}

\begin{tabular}{|c|c|c|}
\hline tipo de alcance & fallas de apropiación & fallas de coordinación \\
\hline Bienes públicos & $\begin{array}{l}\text { - Limitada oferta de I\&D básica y } \\
\text { - Costratégica } \\
\text { - (descubrimientos pioneros) } \\
\text { - Asimetrías de información en el } \\
\text { - } \text { Carencado de tecnología } \\
\text { tendencias científicas y tecnológicas }\end{array}$ & $\begin{array}{l}\text { - Limitación en la oferta de I\&D sectorial } \\
\text { - Escasa prestación de servicios técnicos } \\
\text { - Insuficiente oferta de formación y } \\
\text { - } \quad \text { capacitación en sectores } \\
\text { la falta de inversión en I\&D en campos } \\
\text { relacionados }\end{array}$ \\
\hline
\end{tabular}




\begin{tabular}{|c|c|c|}
\hline tipo de alcance & fallas de apropiación & fallas de coordinación \\
\hline Bienes públicos & $\begin{array}{l}\text { - Escasez de infraestructura de ciencia y } \\
\text { tecnología } \\
\text { - Insuficiente oferta de formación técnica } \\
\text { - } \text { y tecnológica } \\
\text { Grado de inadecuación del sistema de } \\
\text { propiedad intelectual }\end{array}$ & $\begin{array}{l}\text { - Bajo nivel de innovación debido a las } \\
\text { dificultades de coordinación entre } \\
\text { empresas para asumir altos costos fijos } \\
\text { y cuantiosas inversiones } \\
\text { - Duplicación y redundancia de esfuerzos } \\
\text { de I\&D }\end{array}$ \\
\hline $\begin{array}{l}\text { Intervenciones } \\
\text { de mercado }\end{array}$ & 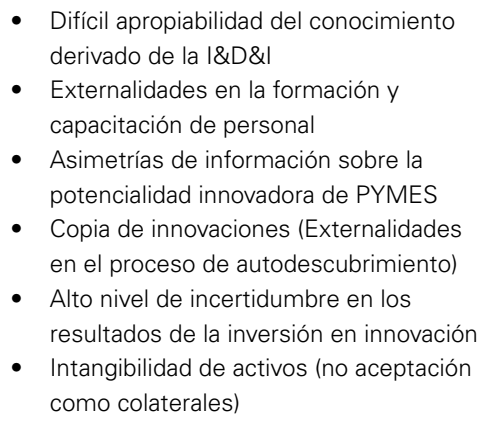 & $\begin{array}{l}\text { - Problemas de apropiabilidad del } \\
\text { conocimiento en determinados } \\
\text { sectores } \\
\text { - Asimetrías de información sobre la } \\
\text { potencialidad innovadora de empresas } \\
\text { en sectores específicos } \\
\text { - Alto nivel de incertidumbre en los } \\
\text { resultados de la innovación en sectores } \\
\text { específicos }\end{array}$ \\
\hline
\end{tabular}

Fuente: elaboración propia a partir de BID $(2014 ; 2016)$.

En síntesis, las fallas de mercado son de dos tipos: de apropiación del conocimiento - generalmente comunes a la mayoría de sectores productivos- y de coordinación -manifestadas esencialmente en sectores productivos específicos-. Dichas fallas se pueden superar con bienes públicos y con intervenciones de mercado, dando origen a cuatro conjuntos de políticas públicas: provisión de bienes públicos a todos los sectores y a sectores específicos; e intervenciones de mercado comunes a los sectores productivos y en sectores o clusters determinados (ver tabla 1).

\subsection{Fallas de sistema}

Se definen, con relación al logro de prioridades clave, como la brecha entre las demandas provenientes de actores y elementos del sistema y la oferta actual disponible. En Colombia, uno de los ejemplos es la situación derivada de la llamada "pirámide invertida» en la que, frente a la demanda latente de formación técnica y tecnológica, la oferta correspondiente es mucho menor que aquella. Otra falla es la débil vinculación de las empresas al sistema nacional de innovación (Teubal, 2008).

\subsection{Fallas de Estado}

Este numeral lo desarrollo sobre la base de la elaboración del caso chileno (Consejo Nacional de Innovación para la Competitividad, 2007). Estas fallas están presentes en cualquier política pública, aunque con mayor incidencia en el caso de la Ciencia, Tecnología e Innovación (CTel).

La «inconsistencia dinámica» se refiere a la dificultad que tiene una autoridad pública para mantener una acción destinada a impulsar la investigación e innovación, debido 
a que generalmente los retornos exceden el respectivo periodo de gobierno. El resultado es que la autoridad pública prefiere realizar inversiones en otros sectores cuyo impacto o retorno se logra a corto o mediano plazo. La «captura de rentas» se da cuando un actor se beneficia de un mecanismo o instrumento de apoyo a la innovación a pesar de no necesitarlo en tanto no existe una falla de mercado que justifique su superación y con frecuencia es el resultado de lobby. Los "problemas de agencia» se refieren a los problemas que surgen cuando en una misma institución cohabitan las funciones de liderazgo de la política de largo plazo y la ejecución de parte o de la totalidad de dicha política, con lo cual, en el momento de evaluación del impacto de la política, se genera una incompatibilidad porque una misma institución es juez y parte.

Existen mecanismos para superar estas fallas de Estado y el diseño institucional mismo apunta a solucionarlas a nivel del sistema.

\section{Diagnóstico del sistema colombiano}

Es evidente que en Colombia, como en muchos países de América Latina, se ha utilizado históricamente, sin suficiente reflexión académica y política, el enfoque conceptual de sistema nacional de innovación, lo cual justifica en cada caso la pregunta sobre la existencia misma de aquel (Velasco, 2015). Empero, en este artículo consideramos útil compartir algunas problemáticas de la dinámica relacionada con la innovación en el país, aunque con una aproximación arriba-abajo.

\subsection{Problemática relacionada con fallas de apropiación del conocimiento}

Este numeral se basa en el contenido del documento borrador de política con corte a junio de 2016 (Colciencias, 2016).

\subsubsection{Insuficientes capacidades para realizar investigación y desarrollo}

Este problema se expresa en la cantidad y calidad de los investigadores y grupos de investigación, en las limitaciones de la infraestructura científica y tecnológica, en la muy reducida inversión pública y privada en I\&D y en el limitado direccionamiento estratégico.

Con relación a la "cantidad y calidad de investigadores, centros y grupos de investigación», de acuerdo al Global Innovation Index, para 2016, Colombia cuenta con 151.9 investigadores equivalentes en tiempo completo por millón de habitantes (Dutta, Lanvin \& Wunsch-Vincent, 2016), ubicándose en el décimo lugar en América Latina, promedio que, comparado con la media de la OCDE de 5826 investigadores por millón de habitantes, sitúa al país en una situación de considerable atraso.

En cuanto a la «financiación de la $\mid \& D$ », hay que resaltar en primer lugar el bajo nivel de inversión: 0.19\% del PIB en 2014, situándose por debajo de países como Brasil, Argentina, México y Chile. El mayor peso en la inversión colombiana lo asume el sector público con más del $54 \%$ durante la última década. Con relación a la ejecución de los recursos, las instituciones de educación superior (IES) y los centros de investigación y desarrollo tecnológico concentraron, en el periodo 2004-2014, el 65.4\% de la inversión en 
$I \& D$, en tanto que las empresas el $23.5 \%$ y las entidades del gobierno nacional el $5.2 \%$ (Observatorio Colombiano, 2015).

Con relación al «bajo direccionamiento estratégico de la $\mid \& D$ », es preciso anotar que hasta hace 10 años se elaboraron planes estratégicos en los programas nacionales de CTel; en adelante, no se renovaron los ejercicios en la mayoría de los casos. En los departamentos se han realizado ejercicios de planeación de CTel que, en general, están vigentes, pero se abordaron con metodologías diferentes y estrictamente centrados en los límites político-administrativos de las entidades territoriales. Hay que subrayar que Colombia solo ha contado con un ejercicio de planeación de largo plazo en CTel realizado en 2006 en el marco de la Visión Colombia II Centenario.

Entre los efectos de este problema, hay que mencionar la dispersión en las líneas de acción y, en general, la concentración de la actividad de Ctel y las brechas entre las regiones. Esto se relaciona también con la dispersión del apoyo público en una gran cantidad de sectores productivos, lo cual en muchos casos ha generado débiles impactos, desperdiciando oportunidades de sinergias y resultando en escasos niveles de diversificación y sofisticación del aparato productivo (Consejo Privado de Competitividad, 2014).

En el ámbito de América Latina, según SCImago (2016), la producción colombiana de publicaciones científicas sigue siendo modesta, aunque es el país que ha tenido una mayor tasa de crecimiento en el periodo 2003-2014, representando en 2014 el 5.8\% del total de publicaciones de la región, ubicándose en el quinto lugar por debajo de Brasil (51\%), México (15.1\%), Argentina (9.79\%) y Chile (8.26\%).

\subsubsection{Insuficiente capital humano para la realización de actividades de ciencia, tecnología e innovación con criterios de calidad y pertinencia}

Este problema se expresa principalmente en la escasez de personal altamente calificado y de formación técnica y tecnológica. La cantidad de personal altamente calificado en Colombia es reducido en comparación con América Latina. En 2013 se graduaron 6.96 doctores por millón de habitantes, mientras que en Brasil o México lo hicieron 70.6 y 43.7 doctores por millón de personas, respectivamente ${ }^{2}$. En el caso de los países de la Organización para la Cooperación y el Desarrollo Económicos (OCDE) se graduaron en promedio, en 2003, 137 doctores por millón de habitantes.

A pesar de lo anterior, el crecimiento de la graduación de doctores en Colombia ha sido positivo en la última década a partir de 2009, alcanzando el nivel más alto en ese año. En cuanto a la calidad de dichos programas, hay que tener en cuenta que, de acuerdo al Sistema de Información de Educación Superior del Ministerio de Educación Nacional (SNIES), solo cinco — equivalente al 2.4\% — contaban con registro de alta calidad en el año 2014.

\subsubsection{Escaso avance en la innovación y el emprendimiento empresarial}

El problema se expresa en que la mayoría de las empresas no innovan: el $73.65 \%$ en el sector de servicios (DANE, 2016) y el $76.86 \%$ en la industria (DANE, 2015). Solo el $0.1 \%$

2. Consultar: $<$ http://www.ricyt.org/indicadores $>$. 
de las empresas de servicios y de la industria, en cada caso, son innovadoras en sentido estricto $^{3}$. En cuanto a las empresas innovadoras en sentido amplio, de ellas, en el sector servicios, logran innovaciones para la propia empresa el 95.9\%, y el 93.8\% en la industria, de las cuales solo un $4 \%$ y un $6.2 \%$, respectivamente, son empresas que innovan para el mercado nacional; es decir, apenas 74 y 105 empresas, en cada caso.

\subsection{Problemática relacionada con fallas de coordinación}

Este numeral se desarrolla sobre la base del avance de la elaboración de la política pública de CTel a junio de 2016 (Colciencias, 2016). El principal problema es la débil coordinación de los actores.

\subsubsection{Débil coordinación de los actores}

El problema de la escasa coordinación de los actores en todos los niveles y entre sectores se manifiesta en duplicidades en las intervenciones de varias instituciones, en el desbalance en la oferta de instrumentos y mecanismos de apoyo a la innovación, y en la carencia de suficiente apoyo en algunos niveles de intervención (por ejemplo, el muy débil apoyo a la gestión de la política: la prospectiva estratégica del sistema).

A nivel de las empresas en el sector industrial, para 2014, el 23.2\% del total de las empresas -innovadoras y potencialmente innovadoras - reportó cooperación con actores del sistema para adelantar actividades de ciencia, tecnología e innovación; y el $4.4 \%$ para I\&D (DANE, 2015). En el sector de servicios, para 2015, las empresas que reportaron cooperación con otros actores fueron el $26.3 \%$ del total de las innovadoras y potencialmente innovadoras; y el $4.3 \%$ para I\&D (DANE, 2016).

\subsection{La gobernanza del sistema nacional de CTel}

La OCDE recomienda la distinción de las responsabilidades entre las unidades que gestionan las operaciones del día a día de aquellas que están comprometidas con una prospectiva estratégica de largo plazo, la planeación estratégica de mediano plazo y el monitoreo del desempeño enfocado a la renovación de la estrategia nacional (OECD, 2014).

Uno de los principales problemas, en la gobernanza, es el severo distanciamiento entre los actores de los ámbitos de competitividad y de ciencia y tecnología (líneas punteadas en el gráfico 1), que debe superarse con el fin de incrementar la efectividad del sistema.

3. Innovadoras en sentido estricto: son aquellas empresas que, en el periodo de referencia de la encuesta de desarrollo tecnológico e innovación, obtuvieron al menos un servicio o bien nuevo o significativamente mejorado en el mercado internacional.

Innovadoras en sentido amplio: empresas que, en el periodo de referencia, obtuvieron al menos un servicio o bien nuevo o significativamente mejorado en el mercado nacional, o un servicio o bien nuevo o mejorado para la empresa.

No innovadoras: son aquellas empresas que, en el periodo de referencia de la encuesta, no obtuvieron innovaciones ni reportaron tener en proceso o haber abandonado algún proyecto para la obtención de innovaciones. 


\section{Gráfico 1: Institucionalidad vigente}

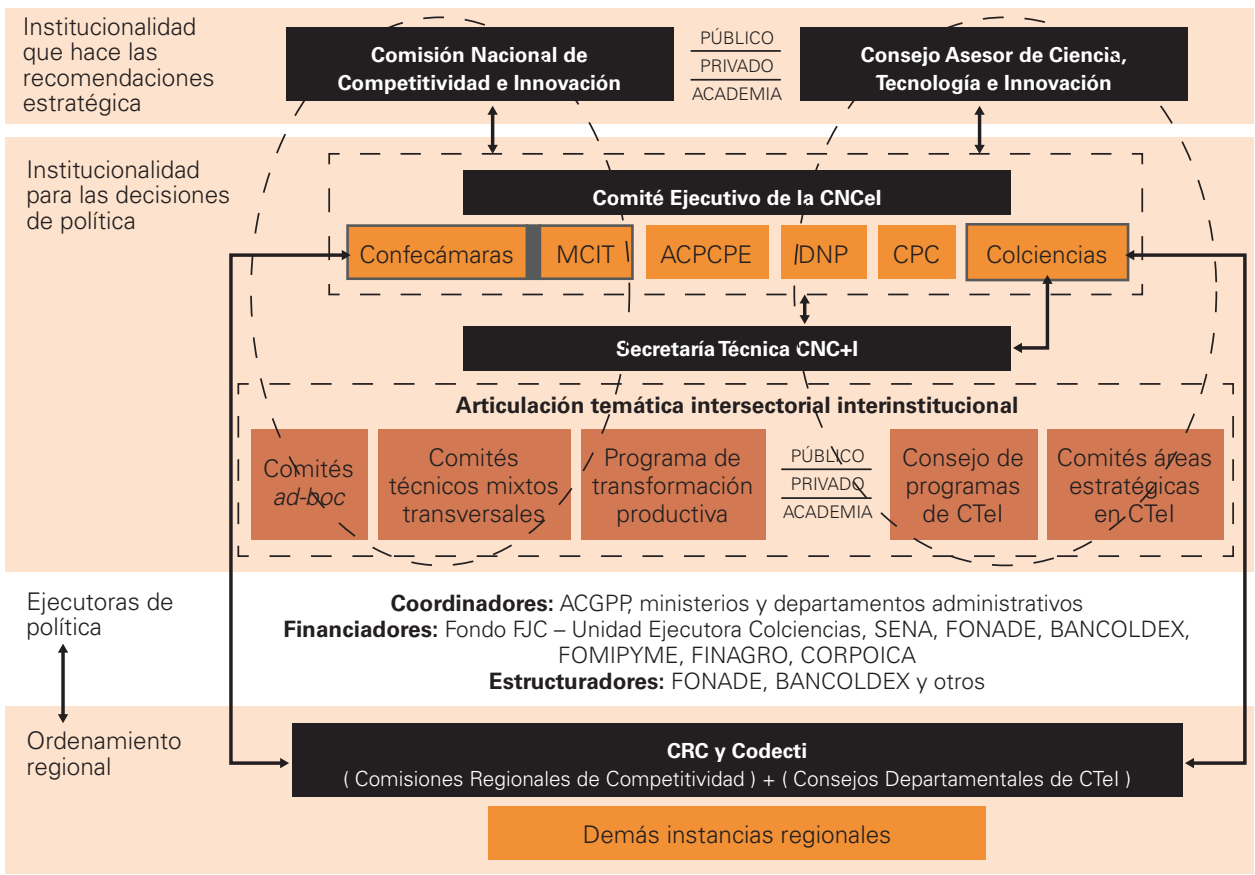

Fuente: Colciencias, 2015.

\subsubsection{Nivel estratégico}

Las funciones sugeridas en este nivel incluyen (Teubal, 2008; OECD, 2014): la definición de las prioridades de CTel relacionadas con los objetivos a largo plazo del país (es el plan de CTel), la definición del portafolio general de la política, el seguimiento y la evaluación del impacto y la definición del financiamiento y el presupuesto general de CTel.

En Colombia actúan dos instancias: la Comisión Nacional de Competitividad e Innovación y el Consejo Asesor de Ciencia, Tecnología e Innovación (ver gráfico 1). La naturaleza de dicha comisión es ser el órgano asesor del Gobierno nacional y de concertación entre este, las entidades territoriales y la sociedad civil en temas relacionados con la productividad y competitividad del país y de sus regiones con el fin de promover el desarrollo económico. Se trata de una instancia de amplia y nutrida participación en los sectores público y privado: presidente de la República, alto consejero presidencial para la Competitividad y Proyectos Estratégicos, director del Departamento Nacional de Planeación (DNP), director de Colciencias, ocho ministerios, director del SENA y 17 representantes provenientes de las regiones, gremios del sector privado, del sector laboral, de universidades, de otras instituciones públicas y personas designadas por el presidente de la República.

En la práctica, en mi opinión, la Comisión Nacional de Competitividad e Innovacion ha demostrado un rol más bien protocolario debido a: 1) la ausencia de una visión compartida e integrada del sistema nacional de CTel; 2) la ausencia de una política de Estado; y 3) la

4. Sobre la base de la revisión de fuentes como actas de la comisión, boletines de prensa y documentos de política. 
falta de cultura institucional de evaluación de impacto y, por ende, de insumos de amplio alcance sobre la base de los cuales realizar ajustes a las políticas. Además, se reúne solo una vez por año.

La misión del Consejo Asesor de CTel es asesorar al Departamento Administrativo de Ciencia, Tecnología e Innovación (Colciencias) en el diseño de la política pública relativa a ciencia, tecnología e innovación. El consejo está conformado por: el director del Departamento, quien lo preside; los ministros de Educación Nacional; Comercio, Industria y Turismo; Agricultura y Desarrollo Rural; Protección Social; y el director del Departamento Nacional de Planeación; el director general del Servicio Nacional de Aprendizaje (SENA); cuatro personas con reconocida trayectoria en el sector académico y científico; y cuatro personas con reconocida trayectoria en el sector productivo.

\subsubsection{Nivel de coordinación}

Desde el punto de vista conceptual, este nivel incluye las funciones de coordinación interministerial y coordinación multinivel entre el centro y los territorios, y entre estos. En este nivel se define el portafolio integrado de mecanismos y servicios de apoyo y se asume la formulación y gestión de programas interministeriales (intersectoriales) que son clave para la resolución de problemas complejos y transversales. Comprende también la definición de presupuestos sectoriales y presupuestos coordinados para la ejecución de los programas interministeriales.

En Colombia, en una primera aproximación, las siguientes instancias e instituciones conforman este nivel: el Comité Ejecutivo de la Comisión Nacional de Competitividad e Innovación, Colciencias, los ministerios, comités técnico mixtos en varias temáticas y los consejos de los programas nacionales de CTel (ver gráfico 1).

El Comité Ejecutivo es el órgano de coordinación y dirección de la Comisión Nacional de Competitividad e Innovación y está integrado por: el alto consejero presidencial para la Competitividad y Proyectos Estratégicos; el ministro de Comercio, Industria yTurismo, el director del DNP; el director del Departamento Administrativo de Ciencia, Tecnología e Innovación (Colciencias); el presidente ejecutivo de la Confederación Colombiana de Cámaras de Comercio; y el presidente del Consejo Privado de Competitividad.

\subsubsection{Nivel de ejecución}

La expresión de este nivel se concreta en agencias ejecutoras sectoriales y a nivel de los territorios, tales como Colciencias, ministerios, SENA, Innpulsa y Bancoldex, entre otras. Actúan las organizaciones que realizan investigación e innovación, como las universidades y los centros de investigación y de desarrollo tecnológico. La normatividad tiene que ver con decretos ministeriales, ordenanzas y acuerdos.

\subsection{Síntesis del diagnóstico de resultados del sistema de CTel}

Tal como se ha evidenciado, la producción científica colombiana, si bien es la más dinámica en Latinoamérica, se ubica en el quinto lugar en la región. La generación de innovaciones es 
muy modesta, lo cual se expresa también, para el año 2015, en 321 solicitudes de patentes para residentes (puesto 49 en el mundo), 1921 solicitudes de no residentes (puesto 28) y 237 solicitudes en el exterior (puesto 56) ${ }^{5}$.

Con relación a la complejidad de las exportaciones colombianas, en 2014, el valor del índice de complejidad económica (ICE) fue de -0,17, ubicándose en el puesto 63 entre 124 países, con una caída de 10 puestos con relación al año anterior y por debajo de México, Panamá, Uruguay, El Salvador, Costa Rica y Brasil, entre los países latinoamericanos mejor situados ${ }^{6}$.

En cuanto a la productividad total factorial (PTF), Colombia ha exhibido un importante rezago en materia de productividad, ya que tuvo un crecimiento anual nulo o negativo durante 12 años, en el período 1991-2015 (DNP, 2016).

\section{Procesos en marcha y enfoques emergentes}

\subsection{Procesos en marcha y metas del país}

En la actualidad, está en proceso la concertación de una política de CTel de mediano plazo hacia el año 2025, cuya visión es convertir a Colombia en uno de los tres países más innovadores de América Latina a través del logro de metas en objetivos de investigación de calidad, innovación y emprendimiento, formación de personal, transferencia de conocimiento y tecnología, apropiación del conocimiento y modernización y adecuación de la gobernanza.

Se aprobó, en agosto de 2016, una política de desarrollo productivo o PDP (CONPES 3866 de 2016), cuyo objetivo general es desarrollar instrumentos que apunten a resolver fallas de mercado, de gobierno o de articulación a nivel de la unidad productora, de los factores de producción o del entorno competitivo, para aumentar la productividad y la diversificación del aparato productivo colombiano hacia bienes y servicios más sofisticados. Entre las metas a destacar hacia el año 2025, se plantea un crecimiento anual de la productividad total de los factores (PTF) del 1\% al 1,3\% y alcanzar, en 2018, por concepto de exportaciones diferentes a las minero-energéticas, la suma de USD 30000 millones.

Entre los temas comunes a las dos políticas, hay que destacar el de la gobernanza, debido a que se ha propuesto la «integración» de los dos "sistemas»: competitividad e innovación y de ciencia, tecnología e innovación (CCTI), en los niveles de orientación prospectiva, coordinación estratégica, ejecución y evaluación.

\subsection{Propuesta sobre la reforma de la gobernanza}

En lo que sigue, sustento variantes, énfasis e innovaciones adicionales a lo propuesto en el actual proceso de formulación de la política.

En el campo de la CTel, es preciso resaltar que la experiencia internacional y la investigación (Teubal, 2008) demuestran que las estructuras de gobernanza más efectivas

5. Consultar: <http://www.wipo.int/ipstats/en/statistics/country profile/profile.jsp?code=CO $>$.

6. Consultar: <http://atlas.cid.harvard.edu/rankings/country/2014/>. 
incluyen instancias decisorias o asesoras del más alto nivel que lideran y aprueban la formulación de políticas de Estado en CTel. Para ello, se cuenta con apoyo técnico y científico en la formulación y concertación de dichas políticas, utilizando nuevos métodos, técnicas e investigación relacionadas con la exploración del futuro, con las tendencias y las experiencias de política de CTel a nivel mundial. Dicho apoyo se expresa, en los países, a través de la actuación de instituciones tales como centros de política o tanques de pensamiento en CTel; por ejemplo, el Centro de Gestión y Estudios Estratégicos de Brasil, STEPI de Corea del Sur, Center for Development Research (ZEF) de Alemania, NISTEP de Japón y CASTED de China, comprendidos entre los 45 primeros reseñados por la Universidad de Pensilvania en 2015 (McGann, 2015); mientras que en Argentina se ha creado recientemente el Centro Interdisciplinario de Estudios en Ciencia, Tecnología e Innovación (CIECTI). Una de las funciones de dichos centros es realizar investigación sobre temas de política de CTel con el fin de fundamentar en conocimiento objetivo las políticas públicas.

\subsubsection{Comisión Nacional de CCTI}

Se sugiere que en su misión se incluya el liderazgo y la aprobación de los planes de Estado de desarrollo productivo y de ciencia, tecnología e innovación, así como la evaluación del impacto de dichos planes. Para ello, se recomienda el apoyo en investigación de un Centro de Pensamiento de Política Pública que puede especializarse en ciencia, tecnología e innovación e incluir, además, desarrollo productivo y TIC. Se destaca la necesidad de separar las funciones de liderazgo en la formulación y evaluación de la política, de la función de ejecución de la misma. La evaluación de impacto de la política se realizará normalmente al finalizar el periodo de gobierno y otros temas específicos por solicitud del alto Gobierno. Esta propuesta incluye la sugerencia de la formulación de planes de largo plazo en desarrollo productivo y un plan de largo plazo en ciencia, tecnología e innovación, con despliegue en cada periodo de gobierno a través de programas de acción y hojas de ruta.

Asimismo, en cuanto toca al fortalecimiento de la capacidad de prospectiva estratégica en estos temas, se recomienda la formulación del plan maestro de un Centro de Política en Ciencia, Tecnología e Innovación y Desarrollo Productivo de carácter público, cuya misión es liderar el diseño y evaluación del impacto de las políticas nacionales de Estado en CTel y desarrollo productivo sobre la base de la investigación y el análisis de las actividades científicas, tecnológicas y de innovación (ACTI), del sector productivo y de los hechos y asuntos con ellas relacionados.

\subsection{Lineamientos para la priorización de apuestas productivas}

En primer lugar, en Colombia, desde hace dos décadas, a pesar de las declaraciones oficiales, sí ha existido una implícita y desordenada política industrial o de desarrollo productivo, y con frecuencia dichas políticas focalizadas no se han guiado por una lógica centrada en superar fallas de mercado, sino en defender rentas de grupos particulares (Meléndez, 2010). En segundo lugar, han existido varios intentos explícitos de focalización —algunos con sólida sustentación técnica-, pero han desembocado en una elevada proliferación de sectores prioritarios a través del Programa de Transformación Productiva y el de Rutas Competitivas 
—al interior de la red Cluster Colombia, entre otros ${ }^{7}$ - reconociendo que su ejecución ha generado importantes experiencias que se deben asimilar. En tercer lugar, frente a la gran incertidumbre en la solidez del actual modelo de desarrollo productivo por sus muy bajos y diferentes niveles de productividad, alto grado de informalidad, débil capacidad para la innovación y por las previsiones de un estancamiento de los precios de los commodities, es perentorio concertar la necesaria focalización a nivel nacional a partir de las regiones y del nivel central.

En el nivel conceptual, a través de los diversos estudios que decantan las experiencias de desarrollo productivo en los últimos 50 años (Koh, 2010), se justifica la adopción de la focalización productiva en los niveles nacional y territorial por sectores, clusters y actividades. En los niveles nacional y territorial, se justifica adoptar el modelo conceptual completo cruzando el tipo de política (horizontal y vertical) con su alcance (bienes públicos e intervenciones de mercado) (Crespi, Fernández-Arias \& Stein, 2014).

Los criterios básicos para la priorización, basados en el marco conceptual expuesto en la sección 2, deben tener en cuenta, en primer lugar, no solo las fallas de mercado, sino las de sistema — vinculadas a unas prioridades - y de Estado; en segundo lugar, las ventajas comparativas latentes y reveladas como línea de base para evaluar el grado de desafio de la ventaja comparativa (Lin \& Chang, 2009); en tercer lugar, la sofisticación vía la innovación; y, en cuarto lugar, con un criterio político y ético, la generación de empleo relacionada con la dinámica de los mercados nacional e internacional (CEPAL, 2012). En síntesis, la priorización se debe concretar en productos (bienes y servicios) de mediano y alto contenido tecnológico por su impacto en la productividad y contribución a la difusión tecnológica, así como en productos que bien pueden ser tradicionales y que requieren un importante volumen de empleo, con demanda en los mercados nacional e internacional, por su impacto en el desarrollo social, constituyéndose en un puente con la política de desarrollo social de gran prioridad en la próxima década a propósito de la construcción de la paz.

\subsubsection{Concertación de prioridades estratégicas}

Para la identificación de los sectores productivos que más aportan al empleo se pueden utilizar "metodologías analíticas»" de tipo estadístico con miras a identificar los sectores productivos que más aportan en el contexto de la región/país en aspectos sociales (como el empleo) o que aportan en aspectos productivos (producción, PIB).

Otro concepto/indicador a utilizar es la "distancia» de los productos que actualmente tienen ventaja comparativa revelada $(\mathrm{VCR})^{9} \mathrm{y}$, en general, de los productos de la canasta exportadora del país, como una medida de cuán factible es readecuar o utilizar las capacidades de las que actualmente dispone el país (Hausmann, 2015).

7. Consultar: $<$ http://www.redclustercolombia.com/ $>$.

8. Sugeridas por la misión de Corea del Sur en el contenido de la Estrategia Nacional de Parques Científicos, Tecnológicos y de Innovación, 2015.

9. Definida como una razón de razones (índice de Balassa): en el numerador la proporción de las exportaciones de un determinado producto sobre las exportaciones nacionales; en el denominador la proporción entre las exportaciones mundiales del bien sobre las exportaciones mundiales totales. 
El nivel de «complejidad» es un concepto/indicador que se constituye en una aproximación a la sofisticación tecnológica con el fin de identificar aquellos productos con un grado importante de ella, dada la comprobación de una correlación positiva entre nivel de complejidad y dinámica del crecimiento del PIB per cápita (Hausmann, 2015).

El concepto/indicador de "oportunidad de ganancia» (Hausmann, 2015) o el indicador de "valor estratégico» (Hausmann \& Klinger, 2007) mide el impacto de los productos que son una especie de "trampolín» para que en el futuro otros productos muy atractivos (complejos) los puedan aprovechar en el proceso de transformación productiva.

El concepto/indicador de "potencial y dinámica de mercado», construido con la base de datos de comercio internacional de UN Comtrade, permite evaluar la demanda actual y potencial de los productos en el mercado internacional. Y para el mercado nacional, se utiliza la información del DATLAS ${ }^{10}$.

Hay necesidad de tener en cuenta que, en la experiencia de varios países de reciente industrialización, ha sido posible "desafiar» la ventaja comparativa e impulsar una transformación productiva hacia sectores o negocios que no demuestran una ventaja comparativa apreciable, pero que tienen efectos potenciales posteriores que dinamizan la economía (caso Korea, Noruega, etc.). Es el caso de la situación de un sector con una ventaja comparativa revelada o latente y con potencial de crecimiento.

Sobre la base de los conceptos e indicadores previamente definidos, se elaboran unos escenarios no excluyentes para la transformación productiva: base, desafío prudente y desafío medio. El "escenario base» se construye con los sectores o productos que poseen VCR, se ubican a corta distancia de otros productos, exhiben un nivel medio de complejidad, importante oportunidad de ganancia y potencial en los mercados nacional e internacional. Se trata de un escenario de diversificación tradicional.

El escenario de "desafío prudente» cuenta con productos o sectores productivos que cuentan con VCR, se encuentran a una distancia media de otros productos, poseen un buen nivel de complejidad, importante oportunidad de ganancia y potencial en los mercados nacional e internacional. Es un escenario que mezcla diversificación y sofisticación. El escenario de "desafío medio" incluye productos o sectores productivos sin VCR, están a una distancia media alta de otros productos, tienen un alto nivel de complejidad, alta oportunidad de ganancia y un potencial medio en los mercados internacionales. En este escenario, se busca una diversificación sofisticada de buen nivel.

La concertación sobre la focalización se logra con la puesta en marcha de un "proceso emprendedor de descubrimiento", en el que participan representantes de la empresa, el Estado, la universidad y la sociedad civil organizada. Los ejercicios técnicos aludidos son insumos para la realización de talleres con la «participación de representantes de la triple o cuádruple hélice», según el caso, y lograr una concertación de las prioridades. Dicho proceso emprendedor de descubrimiento debe generar la visión concertada a largo plazo de la entidad territorial, la región o del país, las prioridades estratégicas en CTel y en desarrollo productivo.

10. Consutar: <http://datlascolombia.com/>. 
La integración "entre el ámbito de la tecnología y la ciencia con el de desarrollo productivo» se logra mediante la identificación de las tecnologías y el conocimiento relacionados con los sectores o productos priorizados. Para ello se sugiere utilizar dos métodos: el primero, mediante el uso de una matriz de concordancia entre tecnologías y sectores productivos con miras a definir las tecnologías clave en cada uno de ellos (Schmoch, 2003; 2008); mientras que el segundo método es el de vínculos algorítmicos con probabilidades, que permite elaborar análisis que relacionan tecnologías y sectores industriales (Travis, 2014). Lo anterior se debe complementar con la opinión de expertos a través de talleres con la participación de empresarios e investigadores.

\subsection{Lineamientos para la focalización en ciencia, tecnología e innovación}

Es deseable que se aborde en lo posible, en un mismo ejercicio, la concertación de la focalización productiva y de la ciencia, tecnología e innovación dada la intensa -aunque no exclusiva - relación de la ciencia con el desarrollo productivo.

Este marco conceptual y metodológico, en primer lugar, aborda la explicación de las interrelaciones y conjunciones entre cuatro ámbitos generales: sociedad, mercado, actividades/cadenas globales de valor (CGV) en sectores productivos, y ciencia y tecnología (ver gráfico 2). En segundo lugar, pretende sugerir algunas combinaciones compatibles de métodos y técnicas que entreguen, a lo largo de un proceso de diálogo informado y transparente entre empresas, centros/universidades y representantes de la sociedad civil, insumos para identificar prioridades en los campos de ciencia e innovación y desarrollo productivo, principalmente.

Gráfico 2: Ámbitos y relaciones básicas hacia la focalización

\section{Sociedad}

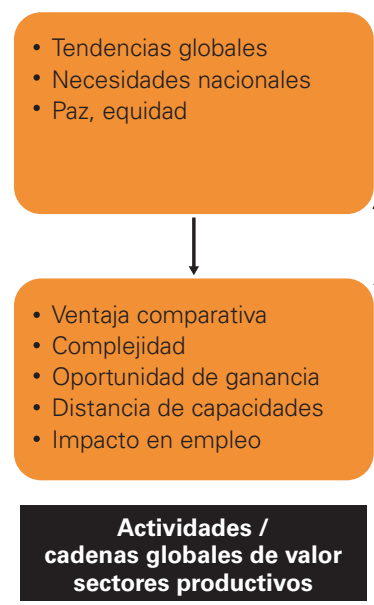
sectores productivos

\section{Mercado}

Tendencias mercado internacional

- Tendencias mercado

nacional

- Países con TLC
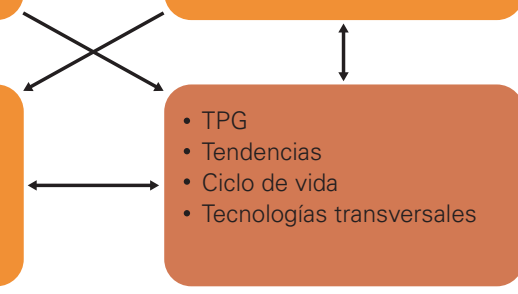

Tecnología y

ciencia

Fuente: elaboración propia (2016).

La dirección del proceso de sustentación y acuerdo de prioridades tiene dos direcciones: arriba-abajo y abajo-arriba. Se espera que este enfoque sea utilizable en los niveles nacional, sectorial y regional. 
En esta ocasión, el punto de partida y llegada es el ámbito de la tecnología y la ciencia —con miras a justificar unas prioridades en este ámbito- que, sin embargo, se integra conceptual y metodológicamente con los ámbitos de las actividades en el marco de las CGV o de sectores, con las demandas de la sociedad y con la dinámica de los mercados internacional y nacional. Las prioridades en tecnología (investigación e innovación) se derivan tanto de las futuras necesidades y vinculaciones con la sociedad y el mercado como de su incorporación en bienes y servicios clave.

En lo que sigue, se detalla la relación entre los ámbitos de ciencia/tecnología y el de actividades/sectores productivos/cadenas globales de valor.

\subsubsection{Tecnología/ciencia - actividades/cadena global de valor/sectores productivos}

Este ámbito es conveniente abordarlo desde el jalonamiento de la demanda y desde el empuje de la oferta. Con relación al primero, es útil seguir las fases generales de: 1) realización de una prospectiva de los cambios en el sector productivo en el futuro y elaboración de un análisis del estado actual de las tecnologías donde se incluya el análisis de las tendencias de las tecnologías de propósito general (TPG), así como de las TIC, la biotecnología y las "tecnologías clave» (nanoelectrónica y fotónica), teniendo en cuenta las necesidades a nivel nacional identificadas en el ámbito de la sociedad con el fin de diferenciar las tecnologías que contribuyen a satisfacerlas; 2) selección de tecnologías clave; 3) formulación de direccionamiento estratégico; y 4) realización de hojas de ruta tecnológica.

Desde la perspectiva del empuje del conocimiento, se realiza un análisis de las tendencias científicas y tecnológicas, identificando tendencias y clusters, ciclos de vida tecnológicos, emergencias a nivel global, regional y del país. Se requiere realizar una evaluación, con el uso de vigilancia o inteligencia tecnológica, de las capacidades de I\&D en el país en cuanto a producción científica y de innovación por temáticas —líneas específicas de investigación - con el propósito de identificar y dimensionar las brechas y fallas de sistema requeridas para lograr las metas de focalización. Entre los métodos y técnicas a utilizar es necesario procesar información secundaria sobre tendencias globales proveniente de fuentes tales como Z-punkt (Alemania), The Dialogue Global Trends ${ }^{11}$, ORBIS ${ }^{12}$ y Scimago, entre otras, además de utilizar diversas herramientas de análisis con información en la web y otras fuentes para complementar técnicas como talleres de expertos y encuestas Delphi (Keun-Ha Chung, 2011). En el análisis del contexto para identificar sus variables clave y sus relaciones, y deduciendo factores de incertidumbre para la construcción de escenarios, se sugiere tomar como referencia ejercicios sectoriales (Nam, Hwang \& Choi, 2011). En los ejercicios de hojas de ruta tecnológica de tipo nacional y temático, puede ser útil adaptar métodos como los utilizados en Corea del Sur (Park, 2015; Yang, 2014).

11. Consultar: <http://globaltrends.thedialogue.org/>.

12. Consultar: <http://espas.eu/orbis/>. 


\section{Conclusiones}

Dada la debilidad del sistema nacional colombiano de CTel, es necesario acoger la recomendación de Velasco (2015) en el sentido de estudiar ámbitos subnacionales y sectoriales dinámicos de innovación como uno de los insumos requeridos para enriquecer la política nacional de CTel y sus mecanismos e instrumentos, buscando acelerar la construcción del sistema nacional y los sistemas sectoriales y regionales. Lo anterior debido al énfasis dado al concepto de «sistema nacional» que, en buena medida, ha pasado por alto la gran diversidad territorial de Colombia, así como su diversificación productiva.

Aunque por limitaciones de espacio no profundizo en el rol del Estado, cabe al menos referir que una reciente investigación demuestra que, en el caso de Estados Unidos, el Estado ha desempeñado en los últimos setenta años un rol muy activo en sectores y actividades de alta tecnología, creando visiones de largo plazo con un enfoque hacia toda la cadena de valor de la investigación/innovación, liderando la inversión en investigación orientada por misión, apoyando la creación de redes descentralizadas entre los actores público y privado, realizando inversiones en las etapas tempranas y creando un portafolio coherente de impulso a la innovación (Mazzucato, 2015).

Entre los componentes clave de una política de Estado de innovación, ciencia y tecnología hay que resaltar los de gobernanza y focalización. El primero debe ser diseñado con miras a superar la falla de inconsistencia dinámica mediante una legitimidad política al más alto nivel, la cual debe estar basada también en una capacidad técnica concretada en un plan de Estado en CTel de largo plazo con la capacidad real de ejercer liderazgo para su formulación, evaluación de impacto y ajuste. El problema de agencia se supera con el ejercicio de una ejecución efectiva de la política por parte de las agencias e instituciones especializadas, apoyadas por un órgano con capacidad de coordinación intersectorial y multinivel, separando de manera clara las funciones de prospectiva estratégica y evaluación de impacto de aquellas de ejecución de la política.

La experiencia internacional, la estrechez fiscal y la equidad justifican la focalización en el campo de la CTel sincronizada con la focalización de una renovada política de desarrollo productivo o nueva política industrial. La focalización se refiere a la justificación concertada entre los sectores público y privado, fruto de un proceso emprendedor de descubrimiento en los niveles nacional y territorial sobre áreas estratégicas que apunten a un objetivo de desarrollo y equidad que, en el caso de Colombia, incluyen objetivos de competitividad productiva y de equidad en el periodo del posconflicto.

Es posible dar cuenta de la disponibilidad de una caja de métodos y herramientas cuantitativas y cualitativas para apoyar procesos participativos de concertación de las focalizaciones de CTel y productivas, destacando la necesidad de fortalecer y afirmar la voluntad política para emprenderlas. Sin embargo, gran parte de la reflexión realizada se basa en la información disponible sobre la economía formal. Se plantea entonces el reto de adaptar y elaborar conceptos y métodos para realizar algo similar con la economía informal que, en el caso de Colombia, representa cerca del $50 \%$ del empleo. Y otro de los principales retos se configura con la definición del aporte de la ciencia y la innovación a la construcción de la paz desde el nivel territorial. 


\section{Acemoglu, Daron \& James Robinson \\ 2012 Por qué fracasan los países. Barcelona: \\ Deusto. \\ Arbeláez, María Angélica \& Mónica Parra \\ 2011 \\ Innovation, $R \& D$ Investment and \\ Productivity in Colombian Firms (IDB \\ Working Papers Series IDB-WP-251). \\ Washington: IDB. Disponible en: <https:// \\ doi.org/10.2139/ssrn.1858064>. \\ CEPAL \\ 2012 \\ Cambio estructural para la igualdad. Una visión integrada del desarrollo. Santiago de Chile: CEPAL.}

\section{Chang, Ha-Joon}

2011

23 Things They Don't Tell You About

Capitalism. Seoul: Penguin Books.

\section{Chung, Keun-Ha \& Cheol-Woo Jeong}

«A new method of key word extraction from foresight topics based on textmining and complexity network analysis». Asian Research Policy, 2 (2), pp. 93-111.

\section{Colciencias}

2016

Política pública de ciencia, teconología e innovación 2015-2025 (avance). Bogotá D.C.: DNP.

\section{Consejo Nacional de Innovación} para la Competitividad

Hacia una estrategia nacional de innovación para la competitividad. (volumen I).

Santiago de Chile: CNIC.

\section{Consejo Privado de Competitividad}

Política de desarrollo productivo para

Colombia. Bogotá D.C.: Consejo Privado de Competitividad.

\section{Consejo Privado de Competitividad}

2015

Informe Nacional de Competitividad 2015-2016. Bogotá D.C.: Puntoaparte Bookvertising.

\section{Crespi, Gustavo}

2010

Impacto del programa de innovación de colciencias: 1995-2007. Washington: BID.

\section{Crespi, Gustavo; Eduardo}

Fernández-Arias \& Ernesto Stein

¿Cómo repensar el desarrollo productivo?

Washington: BID.

\section{DANE}

$2015 a$

Encuesta de Desarrollo e Innovación Tecnológica Industria Manufacturera 2013 2014 - EDIT VI. Bogotá D.C.: DANE.

\section{DANE}

Encuesta de Desarrollo e Innovación Tecnológica Industria Manufacturera 20132014 - EDIT VII. Bogotá D.C.: DANE.

\section{DANE}

2016

Encuesta de Desarrollo e Innovación

Tecnológica Sector de Servicios 2014-2015 - EDITV - Boletín técnico. Bogotá D.C.:

DANE.

\section{Departamento Nacional de Planeación (DNP)}

Dividendo económico de la paz en

Colombia. Bogotá D.C.: DNP.

Departamento Nacional de Planeación (DNP)

2016b Política nacional de desarrollo productivo. Bogotá D.C.: DNP. 


\section{bibliografía}

Dutta, Soumitra; Bruno Lanvin

\& Sacha Wunsch-Vincent (eds.)

2016

The Global Innovation Index 2016:

Winning with Global Innovation. Ítaca,

Fontainebleau y Ginebra: Johnson Cornell

University, INSEAD, WIPO.

\section{Hausmann, Ricardo \&}

Dani Rodrick

2003 Economic Development as Self Discovery. Journal of Development Economics, 72

(2), 425-636. Disponible en: <https://doi. org/10.1016/s0304-3878(03)00124-x>

\section{Hausmann, Ricardo}

The Atlas of Economic Complexity:

Mapping Paths To Prosperity. Cambridge:

Harvard, MIT.

\section{Hausmann, Ricardo \& Bailey}

Klinger

Achieving Export-Led Growth in Colombia.

Cambridge: Harvard.

\section{Koh, Youngsun}

The Korean Economy. Seoul: Korea

Development Institute.

\section{Lin, Justin \& Ha-Joon Chang}

2009 Should Industrial Policy in Developing

Countries Conform to Comparative

Advantage or Defy it? Washington:

WB - Development Policy Review.

Disponible en: <https://doi.org/10.1111

L.1467-7679.2009.00456.x>.

\section{Londoño, J. A.}

2013 High Incomes and Personal Taxation in a Developing Economy: Colombia 1993-2010 (CEQ Working Paper 12). París: Nuffield, EMod-Oxford, Paris School of Economics, Conicet.

\section{Mazzucato, Mariana}

2015

The Entrepreneurial State. Nueva York:

PublicAffaires.

\section{McGann, James}

Think Tanks and Civil Societies Program.

Filadelfia: University of Pennsylvania.

\section{Meléndez, Marcela \&} Guillermo Perry

Industrial Policies in Colombia (IDB

Working Paper 126). Washington: IDB.

Disponible en: <https://doi.org/10.2139/ ssrn.1817239>.

\section{Nam, Sang-Sung; Byung-Yong} Hwang \& Han-Lim Choi

"Foresight study on the energy sector in South Korea». Asian Research Policy, 2 (2), pp. 139-158.

\section{Observatorio Colombiano de Ciencia y Tecnología}

Indicadores de ciencia y tecnología 2014. Bogotá D.C.: Observatorio Colombiano de Ciencia y Tecnología.

\section{OECD}

Draft Synthesis Report on InnovationDriven Growth in Regions: The Role of Smart Specialisation, DSTI/STP/TIP. París: OECD.

\section{OECD}

2012a Public Governance Reviews: Colombia Implementing Good Governance. París: OECD.

\section{OECD}

2014b Reviews of Innovation Policy: Colombia. París: OECD. 


\section{bibliografía}

Park, B. W.

2015

Korean Technology Roadmap. Seúl: STEPI.

\section{Remes, J.}

2015

Productivity, Competitiveness, and Growth in the 21st Century. San Francisco:

McKinsey Global Institute.

\section{Schmoch, Ulrich}

Linking Technologies Areas to Industrial

Sectors. París: Fraunhofer ISI, OST, SPRU.

\section{Schmoch, Ulrich}

2008

Concept of a Technology Classification for Country Comparisons. Ginebra: WIPO Fraunhofer ISI.

\section{Teubal, Morris}

Direct Promotion Of 'Commercial' Innovation (Ci) In Least Developed Countries (LDCs): A Systems Evolutionary Perspective (S/E). Ginebra: UNCTAD Background Paper No. 6.

\section{Travis, Lybbert}

2014

"Getting patents and economic data to speak to each other: An 'algorithmic links with probabilities' approach for joint analyses of patenting and economic activity». Research Policy, pp. 530-542. Disponible en: <https://doi.org/10.1016/j. respol.2013.09.001>.

\section{Velasco, Diana}

2015

Innovation Systems in Developing

Countries: A Top-Down and Bottom-Up Approach to Studying the Colombian National System of Innovation and the Coffee, Flower and Sugarcane Production Chains. Edinburgo: The University of Edinburgh.

\section{Villaveces Cardozo, José Luis}

Modernidad y ciencia en Colombia. Bogotá D.C.: COLCIENCIAS.

\section{Yang, M. C.-L.}

2014 «Procedural characteristics of the 4 th Korean technology foresight». Foresight, 16, pp. 198-209. Disponible en: <https:// doi.org/10.1108/FS-11-2012-0087>

Fecha de recepción: 29/02/2016

Fecha de aceptación: 02/12/2016 